\title{
New models for the evolution of central stars of planetary nebulae: Faster and Brighter
}

\author{
Marcelo M. Miller Bertolami \\ Instituto de Astrofisica de La Plata, CONICET-UNLP, Paseo del Bosque s/n, \\ (B1900FWA) La Plata, Argentina. email: mmiller@fcaglp.unlp.edu.ar
}

\begin{abstract}
The post-asymptotic giant branch (AGB) phase is arguably one of the least understood phases of the evolution of low- and intermediate- mass stars. The recent post-AGB evolutionary sequences computed by Miller Bertolami (2016) are at least three to ten times faster than those previously published by Vassiliadis \& Wood (1994) and Blöcker (1995) which have been used in a large number of studies. This is true for the whole mass and metallicity range. The new models are also $\sim 0.1-0.3$ dex brighter than the previous models with similar remnant masses. In this short article we comment on the main reasons behind these differences, and discuss possible implications for other studies of post-AGB stars or planetary nebulae.
\end{abstract}

Keywords. stars: AGB and post-AGB, planetary nebulae: general

\section{Introduction}

In the most simple picture, Planetary Nebulae $(\mathrm{PNe})$ are formed by low- and intermediate-mass stars $\left(M_{\mathrm{ZAMS}} \sim 0.8-6 M_{\odot}\right)$ after the strong stellar winds at the end of the Asymptotic Giant Branch (AGB). At that point, stars contract and heat up, crossing the HR-diagram at constant luminosity before becoming sufficiently hot and bright to ionize the previously ejected material (Shklovsky 1957, Abell \& Goldreich 1966, Paczyński 1970). The formation and detectability of PNe depends strongly on the interplay between two different timescales. The evolutionary timescale of the central star of the PN (CSPN), which provides the ionizing photons, and the dynamical timescale of the circumstellar material (Schönberner et al. 2007). In this manuscript we focus on the evolutionary timescales of the CSPNe. In particular, we will discuss the new results presented by Miller Bertolami (2016) from full stellar evolution computations of the post-AGB and CSPN phases. These new post-AGB models are based on state-of-the-art stellar evolution computations, they include an updated treatment of the AGB microphysics (radiative opacities and nuclear reaction rates) as well as of the macrophysics (convective boundary mixing and mass-loss rates). The models have been calibrated and tested to reproduce several observables during the post-AGB and previous evolutionary phases; e.g. solar radius and depth of the convective zone, width of the upper main sequence, mass range of carbon-rich AGB stars, oxygen abundances of PG1159 stars, semiempirical initial-final mass relationship, C/O ratios of AGB stars and CSPNe - see Miller Bertolami (2016) for details.

\section{New post-AGB/CSPN evolutionary timescales}

As shown in Fig.1, the post-AGB/CSPN crossing timescales $\left(\tau_{\mathrm{cr}}\right)$ computed by Miller Bertolami (2016) are 3 to 10 times shorter those of the sequences of similar remnant mass $\left(M_{\mathrm{CSPN}}\right)$ computed by Vassiliadis \& Wood (1994) and Blöcker (1995). The new post-AGB models also evolve faster when compared at equal initial masses ( $M_{\mathrm{ZAMS}}$; see Fig. 1$)$ or 


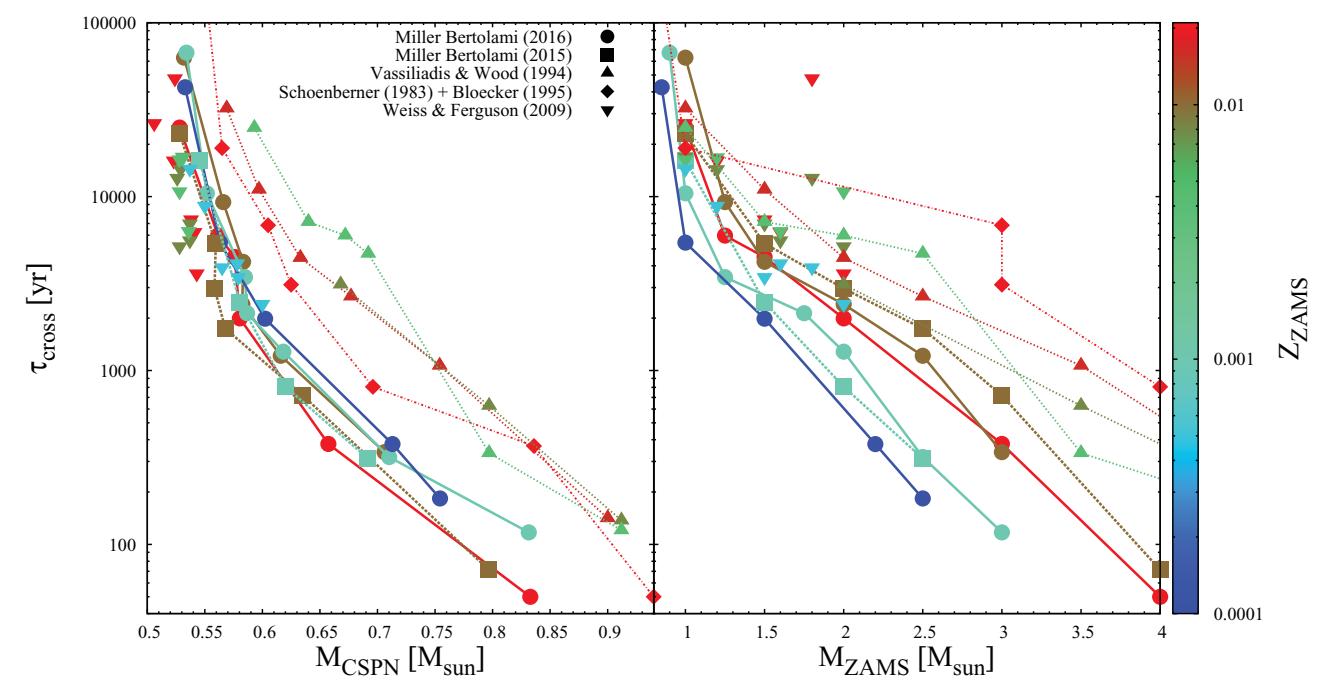

Figure 1. Post-AGB crossing timescales $\left(\tau_{\mathrm{cr}}\right)$ of the sequences presented by different authors. Following Vassiliadis \& Wood (1994) we have defined, and plotted, $\tau_{\mathrm{cr}}$ for all sequences as the time elapsed from $T_{\text {eff }}=10000 \mathrm{~K}$ to the point of maximum $T_{\text {eff }}$ in the HR diagram during the post-AGB evolution. Left Panel: $\tau_{\mathrm{cr}}$ as a function of the final remnant mass $M_{\mathrm{CSPN}}$. Right Panel: $\tau_{\mathrm{cr}}$ as a function of the initial progenitor mass $M_{\mathrm{ZAMS}}$. Color coding indicates the initial metallicities $\left(Z_{\mathrm{ZAMS}}\right)$ of the sequences, as described in the color bar.

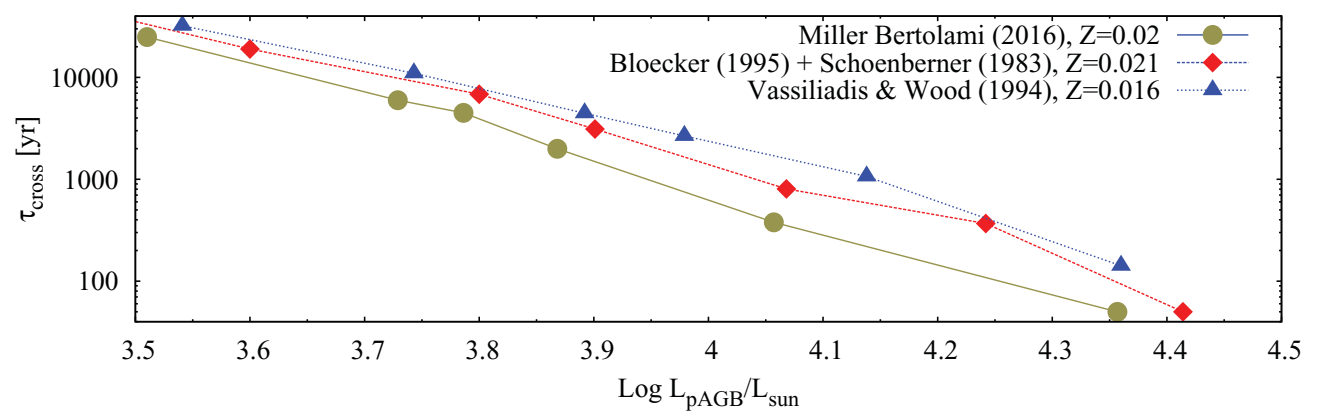

Figure 2. $\tau_{\text {cr }}$ as a function of the post-AGB luminosity for sequences of similar metallicities.

post-AGB luminosities ( $L_{\mathrm{pAGB}}$; see Fig. 2 ). The faster evolution of the new models can be traced back to the adoption of updated microphysics and the inclusion of convective boundary mixing during the thermal pulses on the AGB — see Miller Bertolami (2016) for a detailed discussion. The latter, in particular, leads to the occurrence of third dredge up for lower stellar masses and to carbon (C) pollution of the envelope. In fact, post-AGB timescales become even shorter in the case of the stellar models computed by Weiss \& Ferguson (2009) and Miller Bertolami (2015), which assume a more intense convective boundary mixing during the thermal pulses on the AGB, and consequently have stronger third dredge-up episodes. C-pollution of the envelope increases the luminosity of the burning shell and decreases the critical envelope mass $\left(M_{\mathrm{env}}^{\text {crit }}\right)$ at which the models depart from the AGB. Then, as $\tau_{\text {cr }}$ is mostly determined by the pace at which the hydrogen (H)-burning shell consumes the remaining H-rich envelope, an increase in the C-pollution of the envelope leads to a faster post-AGB evolution. It is worth emphasizing that the value of $M_{\mathrm{env}}^{\text {crit }}$ cannot be arbitrarily set for a given sequence. $M_{\mathrm{env}}^{\text {crit }}$ is mostly set by the H-burning shell luminosity as well as by the composition of the H-rich envelope. As noted 

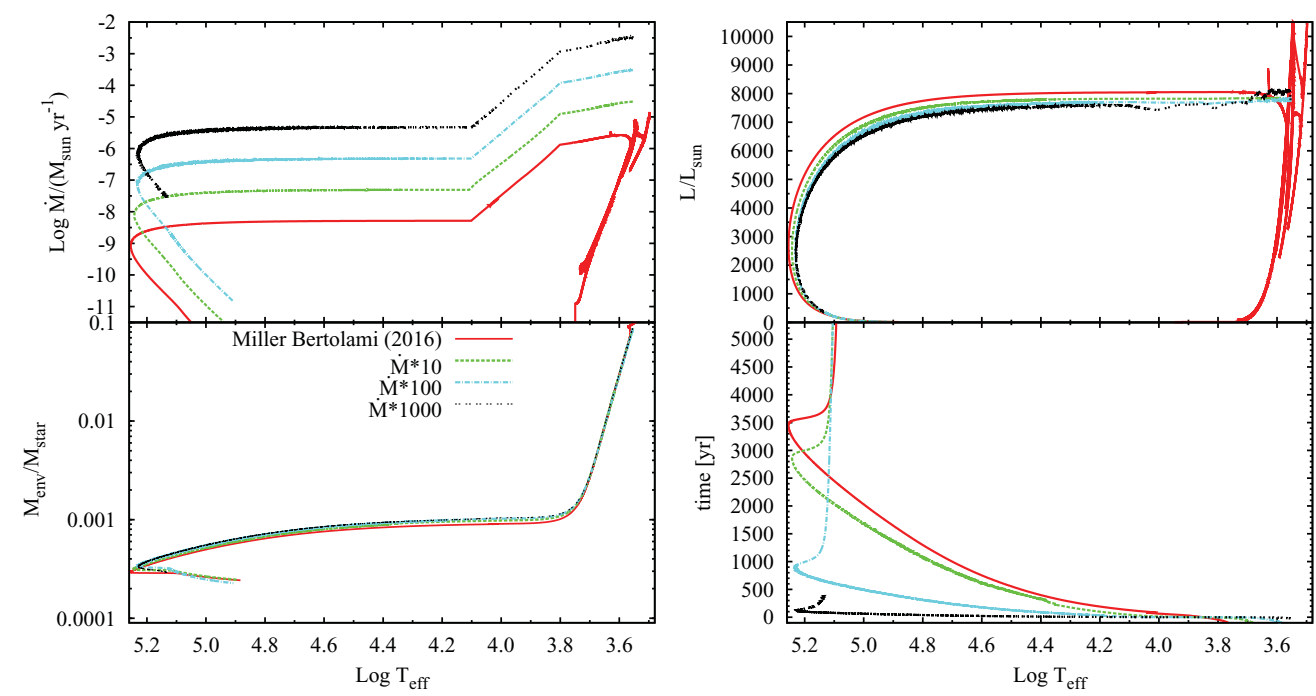

Figure 3. A numerical experiment. Comparison of the late AGB and post-AGB evolution of sequences computed with winds differing by 3 orders of magnitude. The reference sequence is the $M_{\mathrm{ZAms}}=1.25 M_{\odot}, Z=0.001$ sequence from Miller Bertolami (2016).

by Paczyński(1971), H-burning post-AGB models have a very tight relationship between the effective temperature $\left(T_{\text {eff }}\right)$ and the envelope mass $\left(M_{\text {env }}\right)$. The departure from the AGB occurs as soon as $M_{\text {env }}$ is reduced close to these corresponding post-AGB values. In particular, this means that the wind intensity at the end of the AGB plays no role in the determination of $M_{\text {env }}^{\text {crit }}$. This is fortunate, as the intensity of $\dot{M}$ at the end of the AGB, or the early post-AGB, is currently not known. In order to convince the reader of this counter-intuitive fact, we have recomputed the $M_{\mathrm{ZAMS}}=1.25 M_{\odot}, Z_{\mathrm{ZAMS}}=0.001$ sequence presented by Miller Bertolami (2016) artificially increasing the mass loss $(\dot{M})$ after the last thermal pulse by up to 3 orders of magnitude (Fig. 3, upper left panel). As seen in Fig. 3 (bottom left panel) the value of $M_{\text {env }}^{\text {crit }}$ is independent of $\dot{M}$ at the end of the AGB. Only the post-AGB timescales are affected (Fig. 3, bottom right panel) because at high values of $\dot{M}$ the H-rich envelope is mostly reduced by winds, speeding up the evolution. It should be noted, however, that $\dot{M}_{\mathrm{AGB}}$ can affect the post-AGB envelope mass in an indirect way. As $M_{\text {env }}^{\text {crit }}$ is strongly dependent on the luminosity of the sequence, and luminosity does not stay constant during the AGB phase, different $\dot{M}$-values during the whole AGB evolution can change the value of $M_{\mathrm{env}}^{\text {crit }}$ for a given sequence.

\section{Implications and final comments}

Given the significantly shorter timescales, higher luminosities and different initial-final mass relations of the models presented by Miller Bertolami (2016), as compared with the usually adopted tracks from Vassiliadis \& Wood (1994) and Blöcker (1995), one may wonder about the impact of these results for studies that rely on stellar evolution models as inputs. For example, Gesicki et al. (2016) studied 32 PNe from the bulge and obtained CSPNe-black-body temperatures and PNe expansion ages. They find that the CSPNe and progenitor masses derived from the post-AGB models of Miller Bertolami (2016) are in agreement with our current understanding of the stellar formation history of the Galactic Bulge and the white dwarf mass distribution. This was not the case when the tracks of Vassiliadis \& Wood (1994) and Blöcker (1995) were used (Gesicki et al. 2014). 
The fact that, for $M_{\mathrm{ZAMS}} \gtrsim 1.25 M_{\odot}$, the post-AGB timescales of the new models are shorter than those of Vassiliadis \& Wood (1994) and Blöcker (1995) for the same initial masses (Fig. 1, right panel) will have an impact in the understanding and modeling of the Planetary Nebula Luminosity Function (PNLF). Mendez (2016) concludes that with the new stellar models, CSPNe with masses as low as $0.58 M_{\odot}$ are able to create relatively bright $\mathrm{PNe}$ - see also Zijlstra et al. in these proceedings. Consequently, the progenitors of PNe close to the cut-off of the PNLF will be older than previously expected, something that will help to explain the lack of sensitivity of the PNLF cut-off to the age of the harboring population. Due to their shorter post-AGB timescales and larger brightnesses, the new models are also expected to have an impact on the study of the diffuse X-ray emission from the inner regions of planetary nebulae by means of radiation-hydrodynamics numerical simulations - e.g. Steffen et al. (2008) and Toalá \& Arthur(2014). In addition to these results, we can also speculate that the shorter post-AGB timescales of the new models will help to understand the formation of PNe around low mass post-AGB stars e.g. as determined by asteroseismology, see Althaus et al. (2008), Calcaferro et al. (2016) and references therein. Also, shorter post-AGB timescales might help to understand the lack of CSPNe and post-AGB stars in M32 (Brown et al. 2008).

A word of caution, while the new models are to be preferred over the older grids, they are not devoid of uncertainties. In particular, the stellar models of Miller Bertolami (2016) fail to quantitatively reproduce the lifetimes of M- and C-type AGB stars, pointing to a need of a better calibration of convective boundary mixing and AGB-winds.

\section{Acknowledgments}

M3B thanks the IAU and the organizers for a travel grant and the waiving of the registration fee which allowed him to attend this wonderful Symposium. Part of this work was supported by ANPCyT through grant PICT-2014-2708 from FonCyT and grant PIP 112-200801-00940 from CONICET and by a Return Fellowship from the Alexander von Humboldt Foundation.

\section{References}

Abell, G. O. \& Goldreich, P. 1966, PASP, 78, 232

Althaus, L. G., Córsico, A. H., Kepler, S. O., \& Miller Bertolami, M. M. 2008, A $\& A, 478,175$

Blöcker, T. 1995, A\&A, 299, 755

Brown, T. M., Smith, E., Ferguson, H. C., et al. 2008, ApJ, 682, 319-335

Calcaferro, L. M., Córsico, A. H., \& Althaus, L. G. 2016, A\& A, 589, A40

Gesicki, K. \& Zijlstra, A. A. 2007, A\&\&A, 467, L29

Gesicki, K., Zijlstra, A. A., Hajduk, M., \& Szyszka, C. 2014, A $\& A$, 566, A48

Gesicki, K., Zijlstra, A. A., \& Miller Bertolami, M. M. 2016, arXiv:1609.08680

Mendez, R. H. 2016, arXiv: 1610.08625

Miller Bertolami, M. M. 2016, A\& A, 588, A25

Miller Bertolami, M. M. 2015, in: Dufour, P. and Bergeron, P. \& Fontaine, G. (eds.), 19th European Workshop on White Dwarfs, ASP-CS 493, p. 83

Paczyński, B. 1970, AcA, 20, 47

Paczyński, B. 1971, AcA, 21, 417

Schönberner, D., Jacob, R., Steffen, M., \& Sandin, C. 2007, A\&A, 473, 467

Shklovsky, I. S. 1957, in: G. H. Herbig (ed.), Non-stable stars, proceedings from IAU Symposium no. 3, Cambridge University Press,, p. 83

Steffen, M., Schönberner, D., \& Warmuth, A. 2008, A\& A, 489, 173

Toalá, J. A. \& Arthur, S. J. 2014, MNRAS, 443, 3486

Vassiliadis, E. \& Wood, P. R. 1994, ApJS, 92, 125

Weiss, A. \& Ferguson, J. W. 2009, A\& A, 508, 1343 


\section{Discussion}

JoNEs: How applicable do you think these models are for post-CE systems?

Miller Bertolami: We don't know much about the ejection mechanisms in CE systems. But if the ejection of the common envelope is very fast and far from thermal equilibrium then the masses of the H-rich envelopes could be far from the predictions of the post-AGB models computed with steady winds prescriptions like mine. Then the time scale could be very different, so one should be very careful (and skeptical) when using this model for post-CE systems. In short, may be not very much depending on how does the envelope ejection happen.

DE MARCo: What is the new central star mass that makes a PN, judged as the CS that has a transition time of less than 50,000 yrs. What was it for the Vassiliadis+Wood 1994 ?

Miller Bertolami: "Crossing time" (i.e. the time from 7,000 K to maximum Teff in most of my H-burning sequences are below 50,000 yrs. It slightly depends on metallicity a bit but crossing times of less than 50,000 yrs are attained by H-burning sequences with

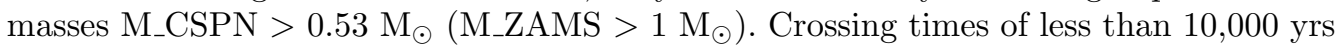
correspond to models with M_CSPN $>0.56 \mathrm{M}_{\odot}\left(\mathrm{M}_{-} \mathrm{ZAMS}>1.2 \mathrm{M}_{\odot}\right)$. "Transition times" (the early post AGB evolution below Teff $<7000 \mathrm{~K}$ ) are ill defined and of a few kyr in my models, decreasing with increasing mass (but very uncertain). Vassiliadis+Wood crossing time scales shorter than 10,000 yrs are only obtained for models with M_CSPN > 0.6 0.63 for H-burning sequences. And their crossing time scales are of a few tens of kyr but with no clear trend in mass or metallicity.

Q: Another crossing-time scale is found from the expansion ages of planetary nebulae. Have you investigated whether the stellar crossing time is consistent with kinematic ages?

ARnABOLDI: You were very quick to show the effects of metallicity of Tau_cross. What are the relevant parameters, in addition to the chemistry that drives this evolution?

STERLING: Do your AGB evolutionary models encounter convergence problems at low envelope masses? Does the envelope mass at the end of the AGB need to be parameterized/ estimated in your models?

Miller Bertolami: Yes, indeed. Also in our models we find a lot of convergence problems as we go to more massive and higher metallicity stars. That is why the models require a lot of baby sitting and are human time consuming to compute. No, the critical envelope mass at which the star departs from the AGB is natural result from the stellar structure computations. It is not artificially chosen. The value of the envelope at the departure from the AGB depends mainly on the structure (mass and radius) of the core and the composition of the envelope (and it is of course affected by the micro physics adopted, e.g. opacity). 